\title{
Microstimulation in Visual Area MT: Effects of Varying Pulse Amplitude and Frequency
}

\author{
Chieko M. Murasugi, C. Daniel Salzman, and William T. Newsome \\ Department of Neurobiology, Stanford University School of Medicine, Stanford, California 94305-3501
}

We have previously shown that perceptual judgements of motion direction are based in part on the activity of direction selective neurons in extrastriate visual area MT (Salzman et al., 1990, 1992). In those experiments, we applied low-amplitude microstimulation pulses $(10 \mu \mathrm{A}, 200 \mathrm{~Hz})$ to clusters of MT neurons whose preferred directions were similar. The effect of microstimulation was to bias the monkeys' choices on a direction discrimination task toward the preferred direction of neurons at the stimulation site. The results suggest that microstimulation generated a directionally specific cortical signal by activating selectively neurons near the electrode tip. To test this notion more directly, we have now examined the behavioral effects of varying current amplitude, current frequency, and electrode position. In the majority of experiments, the directional bias in the monkeys' choices was reduced or eliminated as current amplitude increased to $80 \mu \mathrm{A}$. In addition, $80 \mu \mathrm{A}$ stimulating pulses frequently impaired overall performance as measured by the percentage of correct responses. This decrement in performance indicated that $80 \mu \mathrm{A}$ pulses introduced "noise" into the neural circuitry encoding motion direction, presumably by increasing current spread to activate a larger population of neurons representing all directions of motion. In contrast, increasing current frequency to $500 \mathrm{~Hz}$ (10 $\mu$ A pulses) preserved the directional specificity of microstimulation effects. The precise position of the stimulating electrode also influenced the magnitude of microstimulation effects; in some cases, differences in position on the order of $100 \mu \mathrm{m}$ determined whether an experiment yielded a very large effect or no effect at all. Thus, directionally specific activation of cortical circuitry within MT can be disrupted by increases in current spread or by small changes in electrode position. These observations suggest that the effects of low-amplitude microstimulation depend upon direct activation of a well-localized population of neurons.

[Key words: extrastriate cortex, microstimulation, motion perception, psychophysics, vision]

\footnotetext{
Received July 8, 1992; revised Oct. 19, 1992; accepted Oct. 22, 1992.

We are grateful to Drs. Kenneth Britten and Michael Shadlen for helpful comments and suggestions during the course of this work. We also thank Judy Stein for excellent technical assistance and Drs. S. Celebrini, J. H. R. Maunsell, and E. Zohary for helpful comments on the manuscript. This work was made possible by a grant from the National Eye Institute (EY-5603). C.M.M. was supported by a postdoctoral fellowship from the Natural Sciences and Engineering Research Council of Canada, and C.D.S. is supported by a Medical Student Research Training Fellowship from the Howard Hughes Medical Institute.

Correspondence should be addressed to Dr. William T. Newsome at the above address.

Copyright (C) 1993 Society for Neuroscience $0270-6474 / 93 / 131719-11 \$ 05.00 / 0$
}

The cortical processing of motion signals in primates occurs primarily within a functionally specialized pathway that extends from striate cortex to the cortex of the inferior parietal lobe (Maunsell and Newsome, 1987). The most intensively studied component of this pathway is extrastriate area MT (or V5), an anatomically distinct structure located on the posterior bank of the superior temporal sulcus. Most MT neurons are physiologically defined as direction selective because they respond with an elevated firing rate to motion in a restricted range of directions (Zeki, 1974; Baker et al., 1981; Van Essen et al., 1981; Maunsell and Van Essen, 1983; Albright, 1984). The direction to which a neuron responds optimally is called its "preferred" direction, and neurons preferring different directions of motion are segregated into cortical columns analogous to orientation columns found in striate cortex. The preferred direction of motion varies systematically from column to column such that all directions are represented for each portion of visual space (Albright et al., 1984; Tootell and Born, 1991).

Several lines of evidence indicate that neural activity in MT contributes to motion vision. Lesions of MT selectively impair motion-based visual capacities (Newsome et al., 1985; Newsome and Paré, 1986, 1988; Siegel and Andersen, 1986; Dürsteler et al., 1987; Dürsteler and Wurtz, 1988; Merigan et al., 1991; Vandenbussche et al., 1991), and single neurons in MT encode motion with sufficient sensitivity to account for psychophysical performance in a direction discrimination task (Newsome et al., 1989; Britten et al., 1992). In recent experiments, we used electrical microstimulation to modify the activity of directionally specific circuits within MT while monkeys performed a direction discrimination task. Microstimulation often biased monkeys' judgements of motion direction, causing them to report that motion was in the direction preferred by neurons at the stimulation site. This result could be obtained even when the actual motion in the display was in the opposite direction (Salzman et al., 1990, 1992). These data demonstrated in a particularly vivid fashion a causal link between neural activity in MT and perceptual performance.

In previous microstimulation experiments, we attempted to introduce a directionally specific signal into the cortical circuitry by activating preferentially neurons that had similar directional tuning properties. To accomplish this, we positioned the stimulating electrode within a column in MT and employed weak stimulating currents $(10 \mu \mathrm{A}, 200 \mathrm{~Hz})$ so as to limit current spread as much as possible to neurons within the targeted column. This effort appears to have been largely successful since the behavioral effects of microstimulation were directionally specific, and since the effects were localized topographically to the receptive field of neurons at the stimulation site (Salzman et al., 1992). The primary goal of the present study was to characterize further 
the intracortical localization of the microstimulation effects by examining the influence of different current amplitudes, frequencies, and electrode positions. We anticipated that high current amplitudes should activate directly an array of columns containing various preferred directions and thus reduce the directional specificity of the behavioral effects. In contrast, high current frequencies should not affect current spread and should therefore preserve directional specificity. The present results confirm these hypotheses. In addition, we found that small changes in the position of the stimulating electrode (100-150 $\mu \mathrm{m})$ could dramatically alter the influence of stimulating current on the monkeys' choices. Thus, neural elements near the electrode tip appear to determine the efficacy of microstimulation.

In a second set of experiments, we determined the current amplitude and pulse frequency required to elicit directionally specific effects on psychophysical performance. We found that current amplitudes as low as $5 \mu \mathrm{A}$ (at $200 \mathrm{~Hz}$ ) could produce robust behavioral effects, a figure that compares favorably with threshold current levels for generating muscle contraction via electrical stimulation of motor cortex or the superior colliculus. Using $10 \mu \mathrm{A}$ pulses, we found that pulse frequencies as low as $25 \mathrm{~Hz}$ could produce convincing effects on performance. Effective stimulation frequencies therefore fall well within the response range of the large majority of MT neurons.

A brief report of these results has been published previously (Murasugi et al., 1991).

\section{Methods and Materials}

\section{Subjects}

The experiments were performed in one female and two male rhesus monkeys (Macaca mulatta) weighing between 8 and $11 \mathrm{~kg}$. Each animal was surgically implanted with a head holding device, an eye coil for measuring eye movements, and a recording cylinder that permitted electrode access to visual cortex in the occipital lobe (Evarts, 1966, 1968; Judge et al., 1980).

The monkeys worked for liquid rewards, and their daily water intake was therefore controlled. The animals had unrestricted access to monkey biscuits, and the diet was supplemented with moist monkey treats and fruit. The monkeys were maintained in accordance with the guidelines set by the U.S. Department of Health and Human Services (NIH) Guide for the Care and Use of Laboratory Animals.

All three monkeys had been employed in previous microstimulation experiments and were fully trained on the two-choice direction discrimination described below.

\section{Visual stimuli}

Visual stimuli were controlled by a PDP 11/73 computer and displayed on the face of a large CRT monitor placed $57 \mathrm{~cm}$ in front of the monkey. During the experimental sessions, the monkey discriminated between two directions of stimulus motion in several hundred test trials. The visual stimuli were dynamic random dot displays described in previous publications (Newsome and Pare, 1988; Britten et al., 1992; Salzman et al., 1992). This display consisted of limited-lifetime (20-30 $\mu \mathrm{sec}$ duration) random dots that disappeared and reappeared at different locations in the display. The strength of the motion signal in the display was varied from trial to trial by changing the proportion of dots carrying an unidirectional motion signal. This proportion was determined by the probability that a disappearing dot would be replaced with a "partner" dot at a fixed offset in space and time. We refer to the proportion of unidirectional dots as the "percentage correlated dots" in a given stimulus. For example, " $0 \%$ correlated dots" is a stimulus in which there is no unidirectional motion signal; this display contains motion evenly distributed among all speeds and directions. At 50\% correlation, half the dots carry a unidirectional motion signal while the remaining half provide the masking motion noise. In a 100\% correlated display, all dots are plotted with a constant spatial and temporal offset; this is the maximum strength motion signal achievable with our display.

\section{Electrophysiological recording and microstimulation}

The recording, stimulation and behavioral procedures were identical to those described in previous publications (Salzman et al., 1990, 1992). The electrode was advanced into cortex with a hydraulic microdrive attached to the recording cylinder. We used tungsten microelectrodes that were coated with parylene and had an exposed tip of $20-30 \mu \mathrm{m}$ length (Micro Probe Inc.; 0.5-1.5 M $\Omega$ at $1 \mathrm{kHz}$ ). The electrode was inserted through a transdural guide tube, and multiunit signals from the electrode were amplified, displayed on an oscilloscope, and played over an audio monitor. We identified MT by its topographic map of visual space and by the characteristic direction selectivity and receptive field size of its neurons.

Microstimulation was applied through the same tungsten microelectrodes used to record the activity of MT neurons. To facilitate the passage of stimulating current, we plated the electrode tip with gold so as to reduce the impedance to less than $1 \mathrm{M} \Omega$. We stimulated selected sites in MT using a biphasic pulse generator (cathodal pulse leading) in series with an optical stimulus isolation unit. Each phase was $0.2 \mathrm{msec}$ in duration, and a $0.1 \mathrm{msec}$ interval separated the cathodal and anodal phases. In the various experiments reported in this article, pulse amplitude ranged from 2.5 to $80 \mu \mathrm{A}$, and pulse frequency varied between 12.5 and $500 \mathrm{~Hz}$.

\section{Behavioral paradigm and selection of stimulation sites}

For all experiments, the monkey sat in a primate chair with its head immobilized; the chair stood inside a frame of magnetic field coils that was part of the scleral search coil apparatus used to measure eye movements (Robinson, 1963; Fuchs and Robinson, 1966).

Upon electrode entry into MT, we searched for a microstimulation site by listening to the multiunit responses of MT cells to moving random dots and to a bar of light while the monkey fixated a light-emitting diode (LED). We advanced the microelectrode in increments of 100 $150 \mu \mathrm{m}$ and characterized physiological properties at each electrode position. A "stimulation site" was defined as a stretch of cortex containing neurons with similar receptive field locations, preferred directions, and speeds for at least $200 \mu \mathrm{m}$ of electrode travel. Small differences in physiological properties at different locations within a stimulation site were tolerated. For example, a small shift in preferred direction, on the order of $45^{\circ}$, did not cause us to reject the possible stimulation site; instead, we frequently would set up the psychophysical paradigm so that the preferred direction within the context of the psychophysical task was half way between the preferred directions measured within the site. Stimulation sites that met our criteria were relatively easy to find since MT neurons are organized in a columnar fashion according to their physiological properties (Albright et al., 1984; Tootell and Born, 1991). We were able to measure responses only along the axis of electrode movement, and thus the physiological characteristics of neurons outside the direct path of electrode travel were unknown.

Once a microstimulation site was chosen, we superimposed the neuronal receptive field onto the visual stimulus aperture by requiring the monkey to fixate an appropriately located LED (Fig. $1 A$ ). In addition, the size of the stimulus aperture was adjusted to match the size of the receptive field. On each trial, the monkey discriminated the direction of motion of a random dot stimulus that appeared within the aperture. The motion signal was either in the preferred direction of the neurons at the stimulation site or in the direction $180^{\circ}$ opposite to the preferred (null direction).

To perform the discrimination, a monkey viewed the random dot display for one second while foveating the fixation point (FP, Fig. 1 A). After this inspection period, the fixation point and random dot display vanished and two LEDs appeared, corresponding to the two possible directions of motion (Pref LED and Null LED, Fig. 1A). The monkey then indicated its choice of motion direction by making a saccadic eye movement to one of the two LEDs. Microstimulation, when present, was applied during the one second presentation of the visual display (Fig. 1B).

Experiments typically included trials at four different correlation levels, including $0 \%$ correlation. At correlation levels greater than $0 \%$ correlation, half the trials contained motion in the preferred direction of the neurons, and half in the null direction. The monkey received a liquid reward for each correct choice. A "correct" answer is not possible at $0 \%$ correlation since motion in the display is entirely random; on these trials the monkey was therefore rewarded randomly with a probability of 0.5 . All trial types occurred in random order, and the reward con- 
tingencies were independent of the presence or absence of microstimulation. Choices in favor of the preferred direction of the neurons will hereafter be referred to as "preferred decisions" and those made in the opposite direction will be called "null decisions."

Experimental results presented in this article address two issues: (1) the degree of intracortical localization of microstimulation effects, and (2) the threshold current amplitude and frequency required to elicit convincing behavioral effects. We conducted three different types of microstimulation experiments in studying these questions; each is described below.

\section{Intracortical localization of microstimulation effects}

Multilevel amplitude or frequency experiments. In these experiments, either current frequency or amplitude was varied while the other parameter was held constant. In most experiments, six amplitude or frequency levels were randomly interleaved within the same block of trials. The amplitude levels were $0,5,10,20,40$, and $80 \mu \mathrm{A}$ (all at $200 \mathrm{~Hz}$ ), and the frequencies tested were $0,25,50,100,200$, and $500 \mathrm{~Hz}$ (all with $10 \mu \mathrm{A}$ pulses). Two hundred trials were presented for each amplitude or frequency level, and a single experiment thus consisted of 1200 trials. For each amplitude or frequency level, the 200 trials were distributed over four correlation levels as described above so as to obtain a psychometric function for every level. We conducted 13 amplitude experiments and 25 frequency experiments. Nineteen of the frequency experiments tested frequencies up to $500 \mathrm{~Hz}$; the remaining six experiments only tested frequencies up to $200 \mathrm{~Hz}$.

Electrode position experiments. These experiments explored the impact of small changes in electrode position on the microstimulation effects. Our previous study indicated that the direction of stimulationinduced choice bias can be reversed when an electrode moves by as little as $250-300 \mu \mathrm{m}$ between neighboring columns that encode opposite directions of motion (Salzman et al., 1992). In the present experiments, we examined whether small changes of electrode position, within a single stimulation sitc, as defincd by the criteria given above, can influence the outcome of a microstimulation experiment. We selected a stimulation site in the usual manner, characterizing the properties of neurons along the path of electrode travel and noting the electrode depth at the beginning and end of the site. We then withdrew the electrode to a point just prior to the beginning of the site and conducted a series of microstimulation experiments, moving the electrode forward 100 or $150 \mu$ between each experiment. Thus, the effect of microstimulation was tested at multiple locations before, within, and after, the physiologically defined stimulation site. The stimulation parameters were held constant at $10 \mu \mathrm{A}$ and $200 \mathrm{~Hz}$ throughout these experiments. Approximately 128 trials spanning four correlation levels were conducted in each experiment, and microstimulation was applied on half the trials. As in all other experiments, the test conditions were randomly interleaved within a block of trials.

\section{Thresholds for the effects}

Single-level amplitude or frequency experiments. To determine the current amplitude and frequency required to produce behavioral effects, we conducted two additional sets of microstimulation experiments. This was necessary because throughout the multilevel experiments, the monkeys employed a behavioral strategy called probability matching that resulted in an excess of decisions favoring the null direction in the nonstimulated condition and at other low current amplitudes or frequencies (see Salzman et al., 1992). In essence, this behavioral strategy worked against the effect of microstimulation at low amplitudes or frequencies, and thereby hampered our ability to detect effects (please see Results for further explanation of this behavioral strategy). To determine thresholds, therefore, we tested one stimulation condition (current amplitude or frequency) against the nonstimulated condition within a single block of trials. Different stimulation levels were tested in each block of trials, and multiple blocks were conducted at a given stimulation site. All amplitude experiments were conducted with pulse frequency set at $200 \mathrm{~Hz}$, and all frequency experiments were performed with an amplitude of $10 \mu \mathrm{A}$. We had found previously that microstimulation effects can dissipate as an experiment progresses (Salzman et al., 1992), so we tested the individual stimulation parameters in ascending order to maximize the probability of observing effects at low current amplitudes and frequencies. The effect of increasing current amplitude was tested at some sites while the effect of increasing current frequency was tested at others.

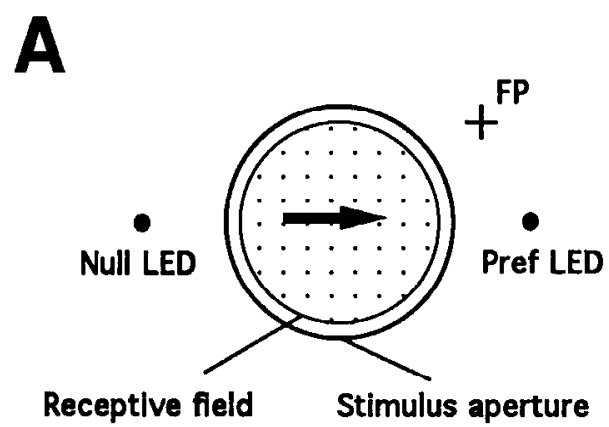

B

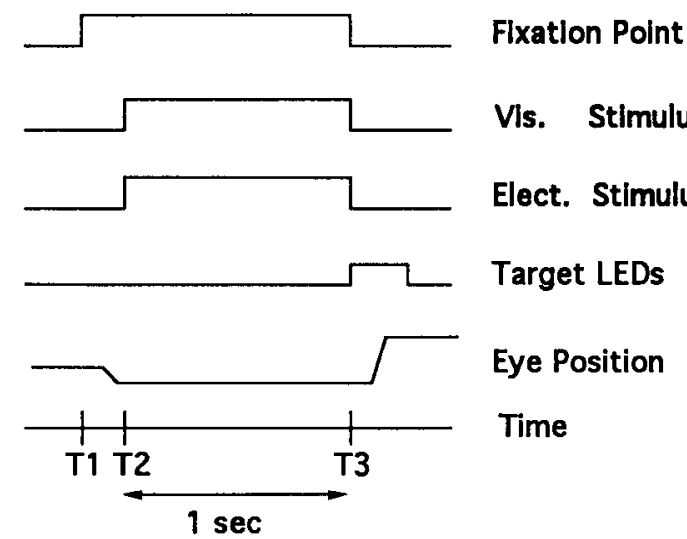

Figure 1. A, The spatial arrangement of the neurons' receptive field (inner circle), visual stimulus aperture (outer circle), fixation point $(F P)$, neuronal preferred direction (arrow), and target LEDs (Pref $L E D$ and Null $L E D$ ) at a hypothetical stimulation site. $B$, The temporal sequence of events in a trial containing microstimulation. Electrical current was applied simultaneously with the presentation of the visual stimulus. Figure adapted from Salzman et al. (1992).

Each single-level experiment consisted of 160 trials -40 each at four correlation levels including $0 \%$ correlation. Half of the trials at each correlation level contained microstimulation, and all conditions were randomly interleaved within the block of trials.

\section{Data analysis}

We analyzed the data from these experiments using logistic regression analysis (Cox, 1970) as described in detail in a prior publication (Salzman et al., 1992). Briefly, logistic regression fits sigmoidal functions to psychophysical data, modeling a monkey's performance as a function of several factors that influence its choices on the direction discrimination task. The psychophysical metric employed in the analysis is the proportion of preferred decisions made by a monkey as a function of the strength and direction of the motion signal. We employed this metric rather than the more commonly used "proportion correct" because we were interested primarily in the effects of microstimulation on the $\mathrm{di}$ rection of a monkey's choices. The logistic model included a free parameter for each factor contributing to the monkey's decisions. Thus, separate coefficients represented a monkey's sensitivity to the strength and direction of motion signals, its inherent choice bias within an experiment, and the possible effects of microstimulation.

The model provided an analysis of two ways in which microstimulation might influence the monkey's performance. First, microstimulation might increase the probability that a monkey will choose a particular direction of motion independently of the actual motion contained in the random dot stimulus. This result would be expected if microstimulation added a directionally specific signal to the cortical circuitry by selectively activating neurons having a common preferred direction. Second, microstimulation might simply degrade the monkey's perfor- 

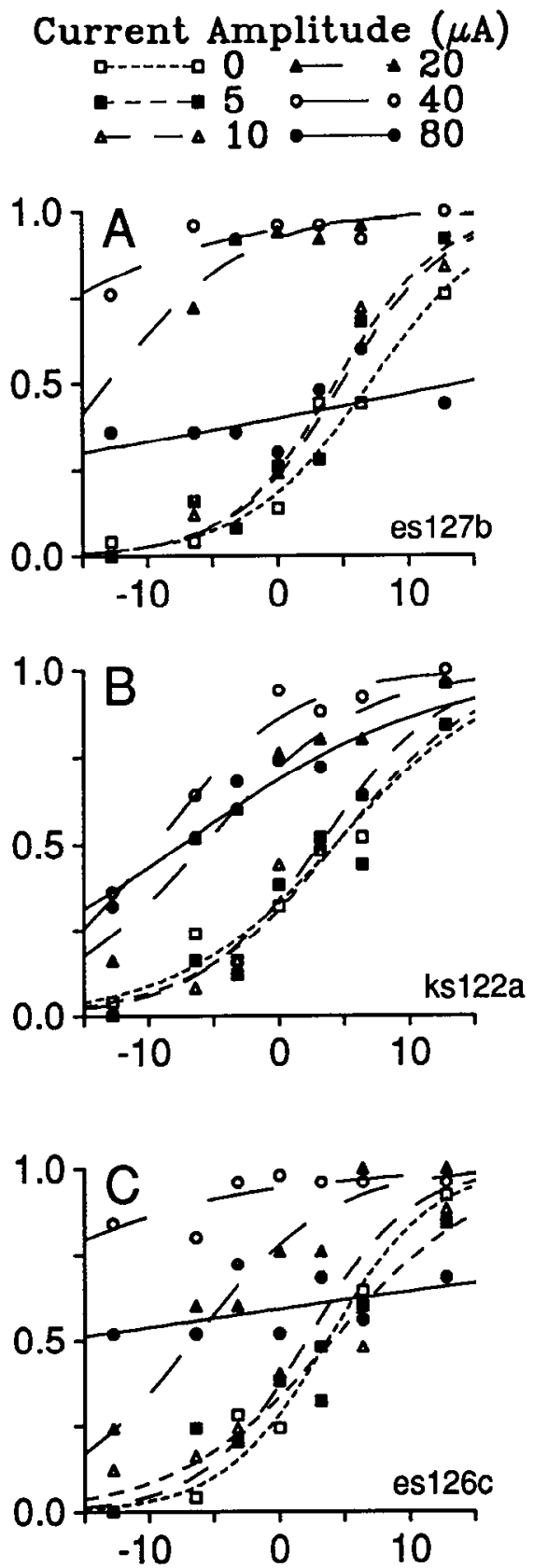

\section{$\%$ Correlated Dots}

Figure 2. Results from three multilevel current amplitude experiments. The stimulating current varied between 0 and $80 \mu \mathrm{A}$; current frequency was held constant at $200 \mathrm{~Hz}$. Each psychometric function depicts the proportion of preferred decisions made by the monkey as a function of the strength of the motion signal. Positive values on the abscissa indicate motion in the preferred direction of neurons at the stimulation site; negative values represent null direction motion. One curve is plotted for each amplitude level tested. Each data point was based on 25 trials, except $0 \%$ correlation, where 50 trials were performed.

mance, causing it to make more incorrect choices without systematically biasing its choices toward either of the two directions. In this case, preferred decisions would increase on null direction trials but decrease on preferred direction trials; the effect of microstimulation on the direction of a monkey's choices would therefore change depending upon the direction and strength of the visual stimulus. This result would be expected if microstimulation added noise to the neural circuitry underlying performance by activating simultaneously neurons that encode widely different directions of motion.

In the multilevel experiments, we examined the effects of applying microstimulation at five current amplitudes or frequencies within the same experiment. We used the logistic function given by Equation 1 to model psychophysical performance; in essence, this function fits separate sigmoidal curves to the data sets for each frequency or amplitude level:

$$
P=\left\{1+\exp \left[-\left(\alpha+\beta \cdot x+\left(\sum_{i=1}^{s} \gamma_{i} \cdot z_{i}+\delta_{i} \cdot z_{i} \cdot x\right)\right)\right]\right\}^{-1},
$$

where $P$ represents the probability of making a preferred decision. The coefficients in the model are $\alpha, \beta, \gamma_{i}$, and $\delta_{i}$, and the variables are $x$ and $z$. $\alpha$ estimates the contribution of choice bias to the monkey's decisions, and $\beta$ represents the influence of the motion signal on the monkey's decisions. $\beta$ is therefore multiplied by $x$, the correlation value of the visual stimulus, and is related to the slope of the psychometric function. Motion in the null direction is entered as negative values of stimulus corrclation sincc null-dircction motion decreases preferred decisions. $\gamma$ and $\delta_{i}$ model the two possible effects of microstimulation for five different stimulation levels $(i=1-5)$, and $z_{i}$ is used to indicate the presence $(z=1)$ or absence $(z=0)$ of microstimulation at a particular level. $\gamma_{i}$ represents a stimulation-induced choice bias toward one of the two possible directions, corresponding to the addition of signal as discussed above. Similarly, $\delta_{i}$ models the addition of noise and is multiplied by $x$ since the effect of adding noise on directional judgements will be different depending upon the visual stimulus direction. If, for example, $\delta_{i}$ is less than 0 , then applying stimulation on null-direction trials will increase preferred decisions since null-direction motion is entered as a negative correlation value.

For the single-level experiments and the electrode position experiments, we used the same logistic model we have employed previously to describe data with low-amplitude microstimulation:

$$
P=\{1+\exp [-(\alpha+\beta \cdot x+\gamma \cdot z)]\}^{-1},
$$

where $P, \alpha, \beta$, and $\gamma$ have the same meaning as in Equation 1. No $\delta$ term is necessary in this model because low-amplitude stimulation rarely adds noise to neural processes encoding motion direction (Salzman et al., 1992).

Using maximum likelihood fitting methodology and appropriate statistical tests, logistic regression provides a quantitative estimate of each coefficient and evaluates whether the value of a given coefficient is statistically different from 0 .

\section{Histology}

Two of the three monkeys employed in this study are alive and being used in related experiments. The remaining animal was killed with a lethal dose of pentobarbital and perfused through the heart with normal saline followed by $10 \%$ formalin. After the perfusion, the brain was removed from the skull, blocked, and equilibrated in a $30 \%$ sucrose solution. Frozen sections $(48 \mu \mathrm{m})$ were cut in the sagittal plane through MT and surrounding cortex. Every fifth section was stained with cresyl violet, and an alternating series of sections was stained for myelinated fibers by the method of Gallyas (1979). Examination of guide tube scars and electrode tracks confirmed that recording and microstimulation took place within the borders of MT as defined by heavy myelination.

\section{Results}

Intracortical localization of stimulation effects. Our previous study suggested that microstimulation of MT influenced judgements of motion direction by restricting direct activation to local clusters of neurons that had similar preferred dircctions. To test this idea further, we applied higher-amplitude stimulating pulses to increase the volume of cortex activated directly (Stoney et al., 1968). We hypothesized that the directional specificity of microstimulation effects would be attenuated by the activation of a more extensive array of cortical columns that encode different directions of motion. In 13 experiments, five current amplitudes were tested together with a "no stimulation" control condition. As described in Materials and Methods, the current 


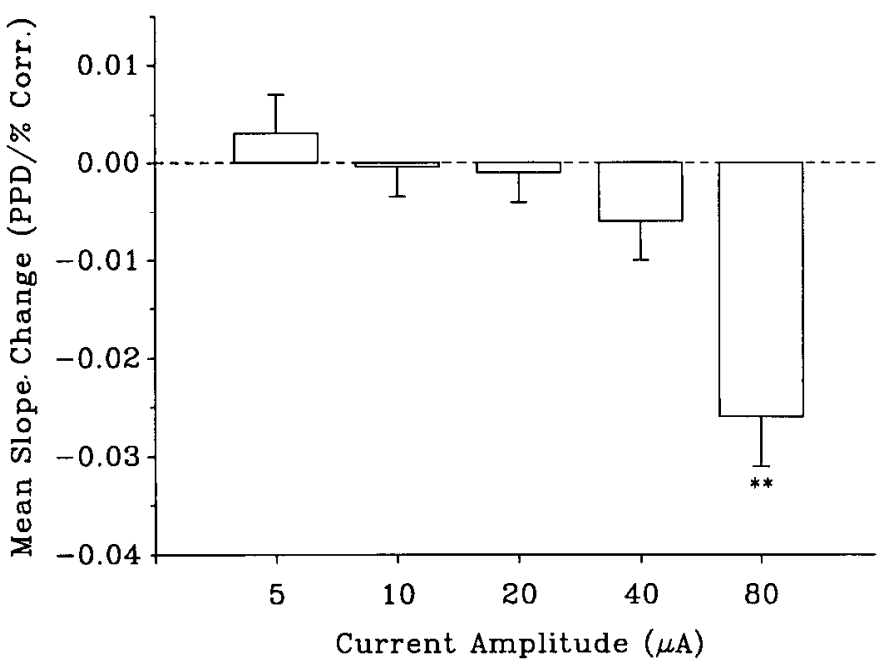

Figure 3. Mean slope change for the "stimulated" psychometric functions relative to the "nonstimulated" functions, plotted for each current amplitude. Data were obtained from 13 multilevel current amplitude experiments. Positive values indicate increases in slope. SE bars denote I SEM, and double asterisks indicate a statistically significant change in slope $(p<0.001, t$ test). The mean change in slope was not significantly different from 0 for any current amplitude except $80 \mu \mathrm{A}$ ( $t$ tests, $p>0.05)$.

levels spanned the range between 5 and $80 \mu \mathrm{A}$, and all test conditions were randomly interleaved within a single block of trials (multilevel amplitude experiments). Figure 2 illustrates results from three of these experiments; each graph plots the proportion of preferred decisions as a function of visual stimulus correlation. Positive correlation values indicate motion in the preferred direction while negative values represent motion in the null direction. Each set of data points and its fitted curve (obtained from Eq. 1) show the results from a single current level.

The data demonstrate two salient aspects of the microstimulation effects. First, in all three experiments, the proportion of preferred decisions made by the monkey increased for current amplitudes as high as $40 \mu \mathrm{A}$, consistent with the notion that microstimulation at these current levels added a directionally specific signal to the neural circuitry in MT (see Materials and Methods). Graphically, the increase in the proportion of preferred decisions is reflected in a leftward shift of the stimulated psychometric functions relative to the nonstimulated function. Higher current amplitudes, especially 20 and $40 \mu \mathrm{A}$, induced larger shifts.

The second striking feature of the data in Figure 2 is the flattened slopes of the psychometric functions obtained with 80 $\mu \mathrm{A}$ stimulating currents. A flattened slope indicates a general reduction in the monkey's sensitivity to stimulus motion since the animal's error rate increased for both preferred and null directions of motion. As described in Materials and Methods, this effect would be expected if microstimulation at the highest current level added noise to the neural circuitry encoding motion direction. In two of the experiments depicted in Figure 2 ( $A$ and $C)$, the psychometric functions for $80 \mu \mathrm{A}$ stimulating currents are nearly horizontal, demonstrating that the monkey could no longer distinguish between preferred- and null-direction motion at any correlation level tested. In some experiments, $80 \mu \mathrm{A}$ currents both flattened the psychometric function and shifted it to the left, suggesting that both signal and noise were added

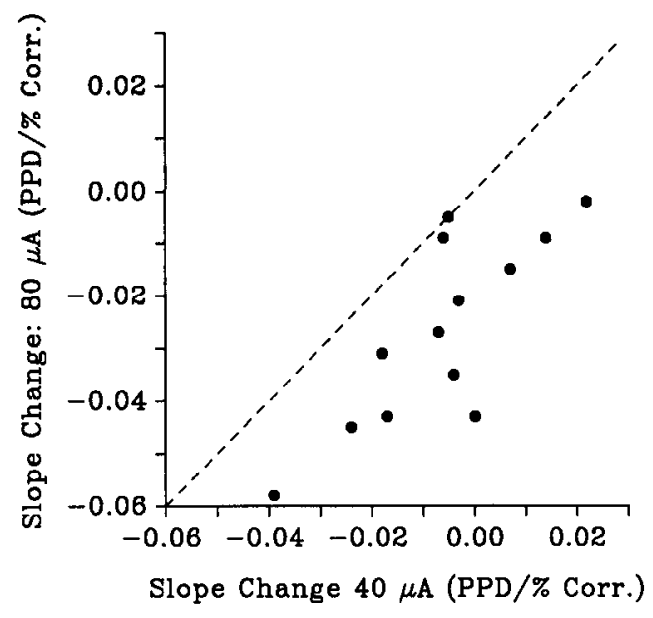

Figure 4. The slope change elicited by $40 \mu \mathrm{A}$ stimulating current is plotted against the slope change obtained with $80 \mu \mathrm{A}$ for each multilevel current amplitude experiment.

to the cortical circuitry. For example, Figure $2 B$ depicts an experiment in which the shift of the psychometric function was quite large at $80 \mu \mathrm{A}(p \leq 0.0001)$, but the flattening of the psychometric function only approached statistical significance $(p=0.11)$. In this case, therefore, $80 \mu \mathrm{A}$ appeared to add mostly signal and only a little noise. Across experiments, the effect of $80 \mu \mathrm{A}$ stimulating currents varied between adding a relatively pure signal (a shift of the psychometric function with no change in slope) to adding only noise (a flattening of the psychometric function with no shift). Overall, $80 \mu \mathrm{A}$ significantly flattened the slope of the psychometric function in 8 out of 13 experiments ( $62 \%$ of experiments; $p<0.05)$.

A quantitative analysis confirmed the impressions derived from inspection of Figure 2. The change in slope at each current amplitude is given by $\delta_{i} / 4$; this value equals the difference in slope of the stimulated and nonstimulated curve at the steepest point on the respective psychometric functions. Figure 3 shows the mean change in slope observed at each current amplitude tested. The mean change in slope did not differ significantly from 0 for any current level between 5 and $40 \mu \mathrm{A}(t$ test, $p>$ 0.15 in all cases). At $80 \mu \mathrm{A}$, however, microstimulation caused a highly significant decrease in mean slope relative to the nonstimulated psychometric functions ( $t$ test, $p<0.001$ ).

Although the mean change in slope for $40 \mu \mathrm{A}$ stimulating currents did not differ significantly from zero, Figure 4 shows that the small slope changes observed with $40 \mu \mathrm{A}$ currents in individual experiments were significantly correlated with the corresponding slope changes observed with $80 \mu \mathrm{A}$ currents $(r=$ $0.79, p<0.01)$. Similar correlations exist for smaller stimulating currents as well ( $p<0.05$ for each current level when compared to $80 \mu \mathrm{A}$ ). The effect of $80 \mu \mathrm{A}$ stimulating currents on the slope of the psychometric function is therefore qualitatively similar to, though much larger than, the effect of smaller currents. Thus, microstimulation can add different amounts of signal and noise depending upon the stimulation conditions. The ratio of signal effect to noise effect is usually high for small current amplitudes, but is frequently low for large current amplitudes.

A reduction in psychophysical sensitivity like that observed with $80 \mu \mathrm{A}$ currents might be expected if the larger currents created lesions of cortex near the electrode tip, but two aspects of the data in Figure 2 rule out this interpretation. First, psy- 

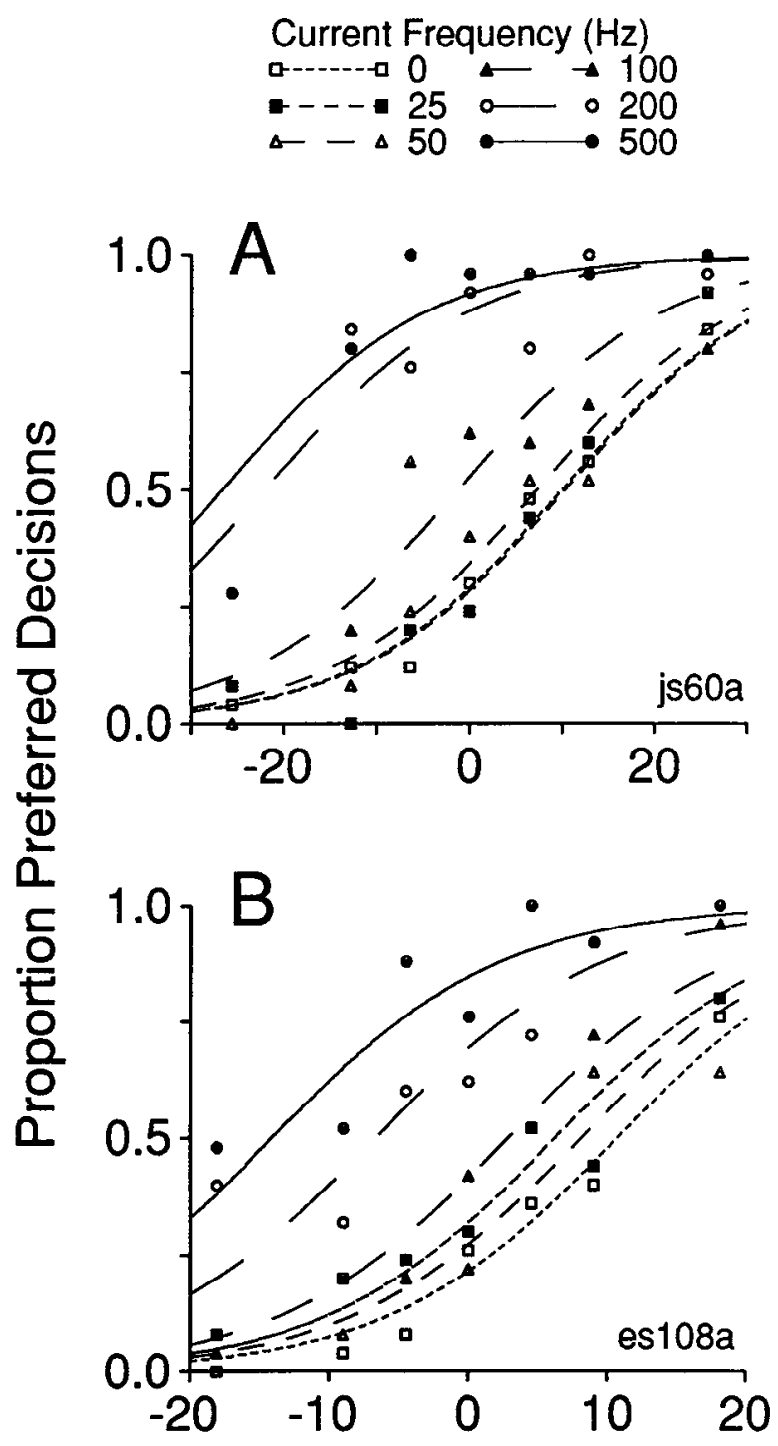

\section{$\%$ Correlated Dots}

Figure 5. Representative results from two multilevel current frequency experiments. Pulse frequency varied from 0 to $500 \mathrm{~Hz}$; pulse amplitude was held constant at $10 \mu \mathrm{A}$. The axes are the same as those in Figure 2. Each curve was fitted to data points obtained at a single frequency level. Each data point is based on 25 trials, except $0 \%$ correlation, where 50 trials were performed.

chophysical performance was normal on nonstimulated trials randomly interleaved within the same experiment; inadvertent lesions should have decreased sensitivity in all trial types within an experiment. Second, the trials with smaller-amplitude currents that were randomly interleaved within the same experiment (e.g., 20 and $40 \mu \mathrm{A}$ ) produced large leftward shifts of the psychometric function throughout the experiment. Thus, neural elements near the electrode continued to influence behavior in a robust, directionally specific manner despite the presence of $80 \mu \mathrm{A}$ currents on other trials within the block. This observation is inconsistent with the notion that local tissue damage is responsible for impaired psychophysical sensitivity on $80 \mu \mathrm{A}$ trials. In addition, the reduction in psychophysical sensitivity did

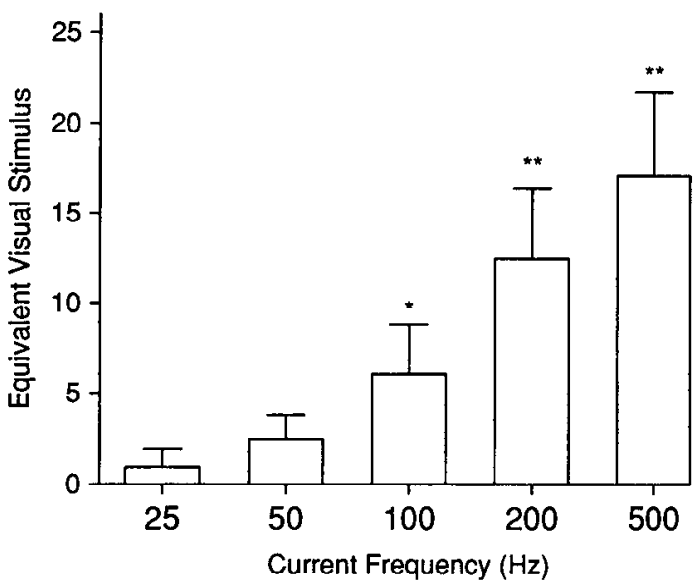

Figure 6. Frequency histogram indicating the mean equivalent visual stimulus for each current frequency level in the 25 multilevel frequency experiments. SE bars denote I SEM. Single asterisks indicate a statistically significant mean effect ( $p<0.05, t$ test); double asterisks indicate highly significant effects $(p<0.01, t$ test).

not appear to be caused by transient inactivation of neurons near the electrode tip. As mentioned above, $80 \mu \mathrm{A}$ commonly shifted the psychometric function as well as flattening it, indicating that signal, in addition to noise, was added to the neural processes encoding motion direction (e.g., Fig. $2 B, C$ ). The addition of signal in the neurons' preferred direction is likely to be mediated by increased neural activity from applying $80 \mu \mathrm{A}$, not inactivation. Indeed, Orbach and colleagues have used optical imaging techniques to show that current amplitudes as high as $100 \mu \mathrm{A}$ can activate neurons in rat striate cortex (Orbach et al., 1991). Thus, it seems unlikely that $80 \mu \mathrm{A}$ inactivates neurons near the electrode tip. The most likely interpretation of our results is that large stimulating currents reduced psychophysical sensitivity by activating an extensive array of columns encoding divergent directions of motion.

In marked contrast to the $80 \mu \mathrm{A}$ data, stimulating frequencies as high as $500 \mathrm{~Hz}$ had little or no effect on the slope of the psychometric function. We fit the data from each multilevel frequency experiment with Equation 1 and found that microstimulation at the highest current frequency $(500 \mathrm{~Hz})$ caused a significant change in the slope of the function in only 1 out of 19 experiments $(p<0.05)$. Moreover, the mean change in slope across experiments was not significantly different from 0 for any frequency level ( $t$ tests, $p>0.3$ for every frequency level). Consequently, we refit the data with a reduced logistic model that lacked the slope terms ( $\delta_{i}$ in Eq. 1$)$.

Figure 5 shows the results of two multilevel frequency experiments in which stimulating frequency was varied from 0 to $500 \mathrm{~Hz}$ while current amplitude was held constant at $10 \mu \mathrm{A}$. Each set of data points shows performance at a single frequency level; the curves within each experiment share the same slope. Above $50 \mathrm{~Hz}$, the primary effect of increasing current frequency was to augment the leftward shift of the psychometric function. We quantified the magnitude of microstimulation effects by calculating the horizontal shift of the stimulated psychometric functions relative to the nonstimulated function, given by $\gamma_{i} / \beta$. We refer to this shift of the psychometric function as the equivalent visual stimulus of a microstimulation effect because it corresponds to the percentage of correlated dots that, if added to the visual stimulus in the neurons' preferred direction, would mimic the behavioral effect of microstimulation. Figure 6 shows 
the mean equivalent visual stimulus observed for each frequency level in the 19 experiments. The mean equivalent visual stimulus was $1.0 \%$ correlated dots at $25 \mathrm{~Hz}$ and increased monotonically for higher frequencies. Thus, increasing current frequency appears to amplify the directional signal within the cortex without degrading the specificity of the signal.

Together, the multilevel current amplitude and frequency experiments suggest that low-amplitude microstimulation directly activates a restricted set of cortical columns. In further support of the notion that microstimulation produces localized activation, we found that precise electrode position can be a critical determinant of the magnitude of microstimulation effects. In these experiments, we advanced an electrode through a cluster of neurons in steps as small as $100 \mu \mathrm{m}$ while stimulating with $10 \mu \mathrm{A}$ currents at $200 \mathrm{~Hz}$. We fitted the data from each experiment with Equation 2 and calculated the equivalent visual stimulus in each case.

Figure 7 shows the results of two sets of electrode position experiments, conducted during two different penetrations through MT. For each set of experiments, the equivalent visual stimulus of the microstimulation effect is plotted as a function of electrode position. Experiments were conducted at electrode positions spaced $100 \mu \mathrm{m}$ and $150 \mu \mathrm{m}$ apart in Figure $7, A$ and $B$, respectivcly. The small arrows indicate the beginning and end of the two stimulation sites, as defined in Materials and Methods; receptive field location and preferred direction are indicated in the diagram in the upper right of each panel. Clearly, small changes in electrode position can have a dramatic impact on the outcome of an experiment. In Figure $7 A$, for example, microstimulation failed to influence the monkey's performance significantly when the electrode was positioned $0.52 \mathrm{~mm}$ or 0.72 $\mathrm{mm}$ into MT (first and third data points; $p>0.05$ ). A large, highly significant result was obtained, however, with the electrode placed at $0.62 \mathrm{~mm}(p \leq 0.0001)$. Figure $7 B$ illustrates a similar set of experiments in which a change in electrode position of $150 \mu \mathrm{m}$ led to a very large change in the magnitude of the microstimulation effect. Some portion of the decrease in effect after electrode movement could be due to a dissipation of the microstimulation effects as trials accumulate. However, the small number of trials in these experiments (128 per experiment) makes that explanation less likely; robust effects of microstimulation are usually evident after 250 trials at a single stimulation site (Salzman et al., 1992).

To summarize the effects at different electrode positions within a stimulation site, we first determined the position within each site that yielded the largest behavioral effect. Across 13 experiments, the "best" position was equally likely to occur in the center of a site, near the edge of a site, or midway between the center and an edge. For each position producing the largest effect, we calculated the percentage decrease in microstimulation effect at the two electrode positions adjacent to the position producing the maximum effect. Across the 13 experiments, the magnitude of the microstimulation effect decreased by an average of $69 \%$ at the adjacent locations. The average distance from the best electrode position to an adjacent location was 140 $\mu \mathrm{m}$. The data therefore demonstrate that precise electrode position within a stimulation site is an important determinant of the magnitude of a microstimulation effect. Consistent with the current amplitude experiments described above, these results suggest that the effects of microstimulation are strongly influenced by neural elements near the electrode tip.

Thresholds for the effect. We next sought to determine the
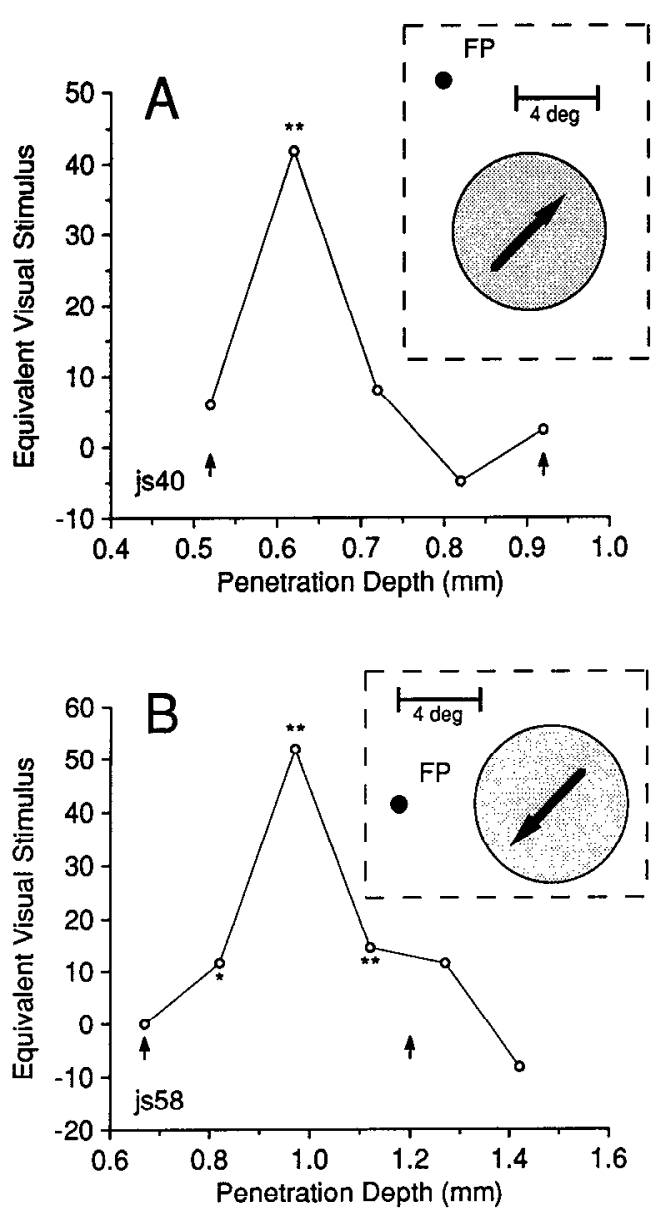

Figure 7. Two sets of experiments in which the effect of microstimulation was measured at regularly spaced electrode positions. The receptive field location (circle), fixation point $(F P)$, and preferred directions (arrows within circle) are shown for each stimulation site within the diagram at upper right. For the experiment depicted in $A$, preferred direction varied from approximately $15^{\circ}$ counterclockwise from the arrow to about $30^{\circ}$ clockwise from the arrow as the electrode advanced from the first position to the last position. Preferred direction varied slightly less in the experiment depicted in $B$. For both sets of experiments, the equivalent visual stimulus is plotted as a function of electrode position. Electrode position is given in millimeters, relative to the point of entry into MT in that penetration. The small arrows indicate the beginning and cnd of what we classified as a single stimulation site. $A, A$ series of five experiments, each separated by $100 \mu \mathrm{m} . B$, A series of six experiments, each separated by $150 \mu \mathrm{m}$. The double asterisks indicate highly significant effects (logistic regression, $p<0.01$ ); the single asterisks represent more weakly significant effects $(p<0.05)$.

threshold values of current amplitude and frequency that could elicit a behavioral effect. In principle, these values could be calculated from the multilevel results presented above. In practice, however, behavioral data from the lowest amplitude and frequency levels within the multilevel experiments are confounded by a pronounced tendency of the monkeys to make choices toward the null direction of motion. This "null bias" is readily evident in the data of Figures 2 and 5; the monkeys consistently made fewer than $50 \%$ preferred decisions at $0 \%$ correlation for low current amplitudes or frequencies. This null bias had the effect of reducing artificially the apparent size of microstimulation effects at low amplitudes and frequencies. We have shown previously that this null bias occurs because the monkeys seek to distribute their choices evenly within a block of trials between the two directions of motion in accordance 


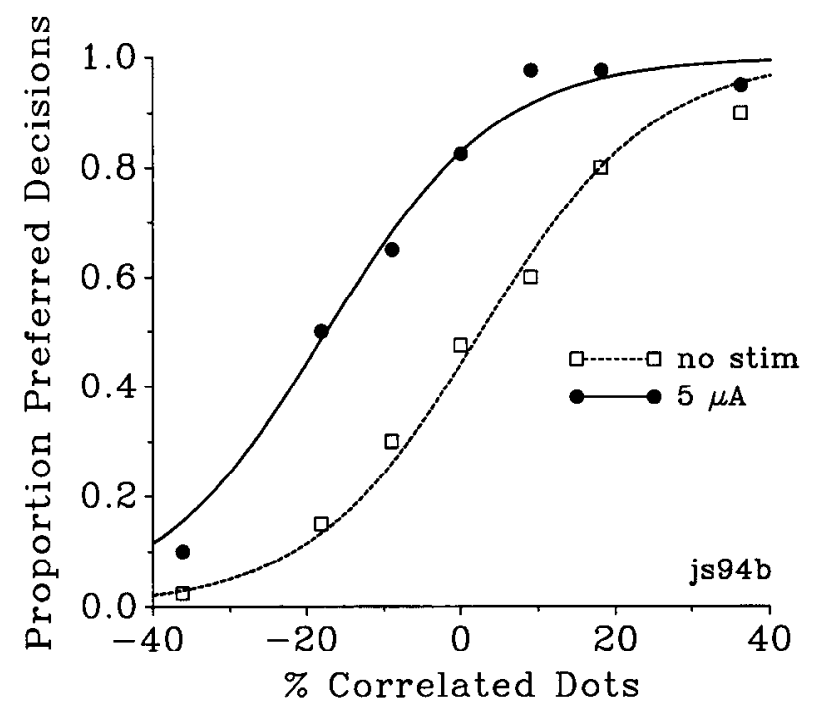

Figure 8. Results from one experiment employing a $5 \mu \mathrm{A}, 200 \mathrm{~Hz}$ stimulating current. The axes are the same as those in Figure 2. Each data point is based on 10 trials except for $0 \%$ correlation, where 20 trials were conducted.

with the probability of being rewarded for each choice (recall that an equal number of trials is presented for the preferred and null directions of motion) (Salzman et al., 1992). Thus, an increase in preferred decisions at high current amplitudes or frequencies will be balanced by an increase in null decisions at low current amplitudes or frequencies. To determine threshold current values, therefore, we performed individually blocked, single-level microstimulation experiments as described in Materials and Methods. We fit the data from each experiment with the same logistic model used in the electrode position experiments (Eq. 2).

Current amplitudes of $2.5,5$, and $10 \mu \mathrm{A}$ were tested in ascending order at 13 stimulation sites in one monkey; the pulse frequency was held constant at $200 \mathrm{~Hz}$. As illustrated in Figure 8 , microstimulation currents as low as $5 \mu \mathrm{A}$ could induce a highly significant increase in choices toward the preferred direction of the stimulated neurons (equivalent visual stimulus $=$ $20.1 \%$ correlated dots; $p \leq 0.0001$ ). Figure 9 shows the mean equivalent visual stimulus for each current level tested in the 13 single-level experiments; $10 \mu \mathrm{A}$ currents affected performance dramatically as expected, but $5 \mu \mathrm{A}$ currents elicited a significant effect as well ( $t$ test, $p<0.05$ ). A current level of 2.5 $\mu \mathrm{A}$ had no measurable effect on the animal's choices. Thus, approximately $5 \mu \mathrm{A}$ is required to elicit microstimulation effects in MT.

To determine the threshold pulse frequency for eliciting behavioral effects, we conducted 14 sets of single-level frequency experiments in the same monkey. Stimulation frequencies of $12.5,25$, and $50 \mathrm{~Hz}$ were tested in ascending order while current amplitude was held constant at $10 \mu \mathrm{A}$. Figure 10 shows the results of an experiment in which a pulse frequency of $25 \mathrm{~Hz}$ elicited a robust effect (equivalent visual stimulus $=13.7 \%$ correlated dots; $p \leq 0.0001$ ). Figure 11 illustrates the mean equivalent visual stimulus obtained at each frequency level. A frequency of $50 \mathrm{~Hz}$ yielded significant effects in 5 out of 14 experiments $(p<0.05)$, and the mean equivalent visual stimulus from these experiments was significantly greater than zero ( $t$ test, $p<0.05$ ). Mean effects at 25 and $12.5 \mathrm{~Hz}$ were weakly

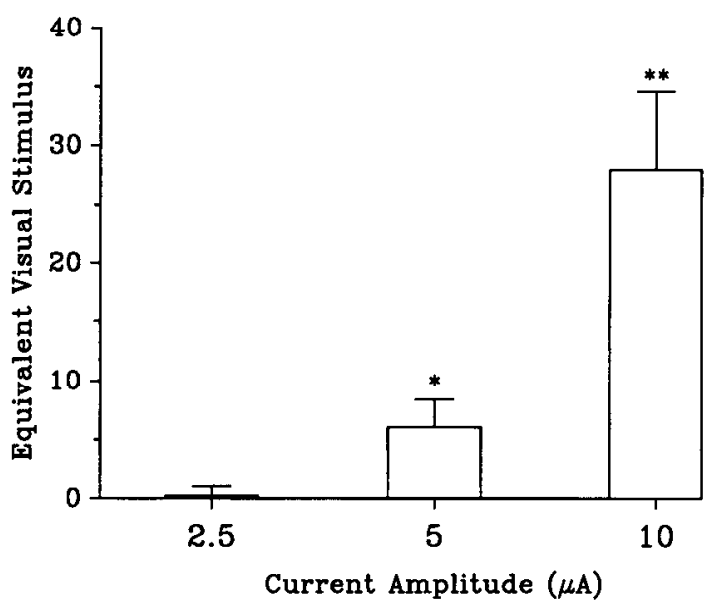

Figure 9. Mean results from all single-level amplitude experiments. The mean equivalent visual stimulus is plotted for each current amplitude. Error bars depict 1 SEM, and asterisks denote the statistical significance of the mean effect $\left(t\right.$ test; $\left.{ }^{*}, p<0.05 ;{ }^{* *}, p<0.01\right)$.

significant $(25 \mathrm{~Hz}: 13$ experiments, mean $=2.8 \%$ correlated dots, $p=0.054, t$ test; $12.5 \mathrm{~Hz}: 11$ experiments, mean $=3.5 \%$ correlated dots, $p=0.040, t$ test). Thus, significant behavioral effects can be induced by a stimulating frequency of $25 \mathrm{~Hz}$, and weak effects may be present for frequencies as low as $12.5 \mathrm{~Hz}$. These frequencies are well within the normal physiological response range of MT neurons (K. H. Britten, M. N. Shadlen, J. A. Movshon, and W. T. Newsome, unpublished observations).

\section{Discussion}

In the present study, we assessed the localization of neural signals arising from microstimulation of MT by varying current pulse amplitude, pulse frequency, and electrode position. These manipulations were directed toward understanding the dependence of microstimulation effects on current spread, rate of neuronal firing, and the functional properties of neurons near the electrode tip.

The most striking result was that increasing the amplitude of current pulses to $80 \mu \mathrm{A}$ frequently decreased the monkeys' sensitivity to the visual stimulus. This loss in sensitivity manifested itself as a flattening of the psychometric functions as illustrated in Figures 2 and 3. The simplest interpretation of these data is that $80 \mu \mathrm{A}$ pulses directly activated neurons in an extensive array of cortical columns such that neurons encoding many, perhaps all, directions of motion were active simultaneously during the discrimination. Nonselective activation of this nature would impair the monkey's performance by obscuring the intricate patterns of directionally specific neural activity that normally exist within MT. Thus, the application of $80 \mu \mathrm{A}$ pulses can be thought of as adding "noise" to the neural processes encoding motion direction. In some experiments, $80 \mu \mathrm{A}$ also added a directionally specific "signal" to the neural representation of motion, as demonstrated by a leftward shift of the psychometric function. When both effects appear in the same experiment, it is reasonable to suppose that neurons encoding a particular direction of motion are activated preferentially, but that neurons encoding other directions are activated to a lesser extent.

Though we did not make direct measurements of current spread in our experiments, our inferences are consistent with 


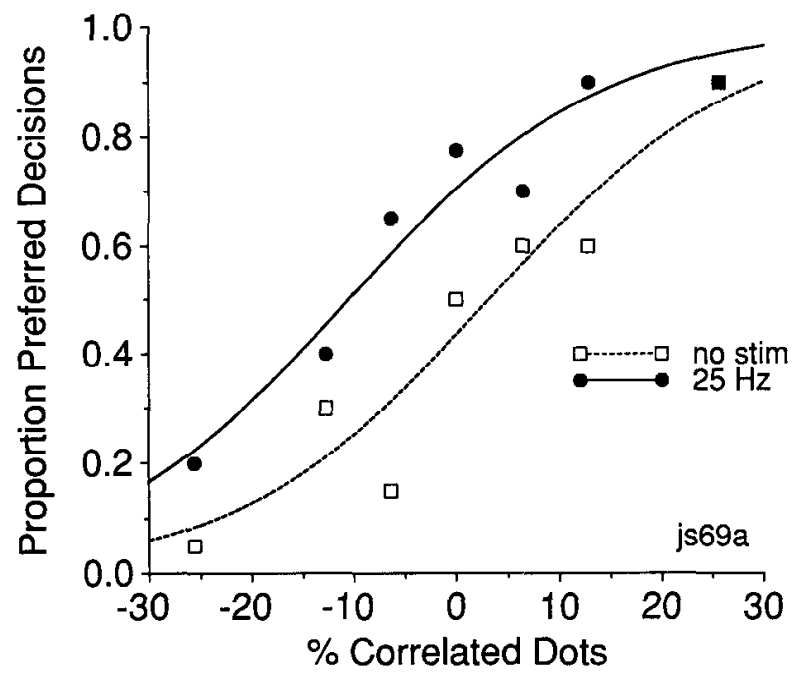

Figure 10. Results from one experiment employing a $10 \mu \mathrm{A}, 25 \mathrm{~Hz}$ stimulating current. The axes are similar to those in Figure 2. Each data point is based on 10 trials except for $0 \%$ correlation, where 20 trials were conducted.

evidence obtained in primate motor cortex. Stoney et al. (1968) estimated that a single $10 \mu \mathrm{A}$ cathodal pulse can excite neurons within approximately $85 \mu \mathrm{m}$ of an electrode tip ( $95 \%$ confidence limits at 70 and $120 \mu \mathrm{m}$, respectively). This figure is consistent with estimates of current spread made independently by Tootell and Born using 2-deoxyglucose techniques in conjunction with a microstimulation regime nearly identical to our own (Tootell and Born, 1991). Extrapolation from data gathered by Stoney and colleagues indicates that increasing current amplitude to 80 $\mu \mathrm{A}$ nearly triples the effective stimulation radius to approximately $250 \mu \mathrm{m}$, potentially resulting in an almost 25-fold increase in the volume of cortex activated directly by the stimulating current. Clearly, this large increase in current spread could excite numerous cortical columns, each encoding a different direction of motion.

Increases in the frequency of stimulating pulses had little or no effect on the slope of the psychometric function in our experiments. Rather, increasing the stimulation frequency up to $500 \mathrm{~Hz}$ (for a constant current amplitude of $10 \mu \mathrm{A}$ ) amplified monotonically the leftward shift of the psychometric function (Figs. 5, 6). Thus, stimulation frequency altered the intensity of the directional signal within the cortex without adding detectable amounts of noise. This enhanced directional signal could have been generated by an increase in transsynaptic activation which results from application of multiple stimulating pulses (Jankowska et al., 1975; Asanuma et al., 1976), and/or by increasing the firing rate of activated neurons (though neurons do not necessarily fire action potentials that follow precisely the frequency of a train of stimulating pulses; E. H. Deyoe, personal communication). The preservation of directional specificity observed at high stimulating frequencies implies that transsynaptic activation within MT was restricted to neurons having similar preferred directions. Transsynaptic activation could also have spread to other cortical areas connected to MT. Local circuits of neurons sharing similar physiological properties have been shown to exist between orientation columns in striate cortex (Ts'o et al., 1986; Ts'o and Gilbert, 1988; Gilbert and Wiesel, 1989), and analogous circuits may therefore be present within MT or between MT and other cortical areas comprising the

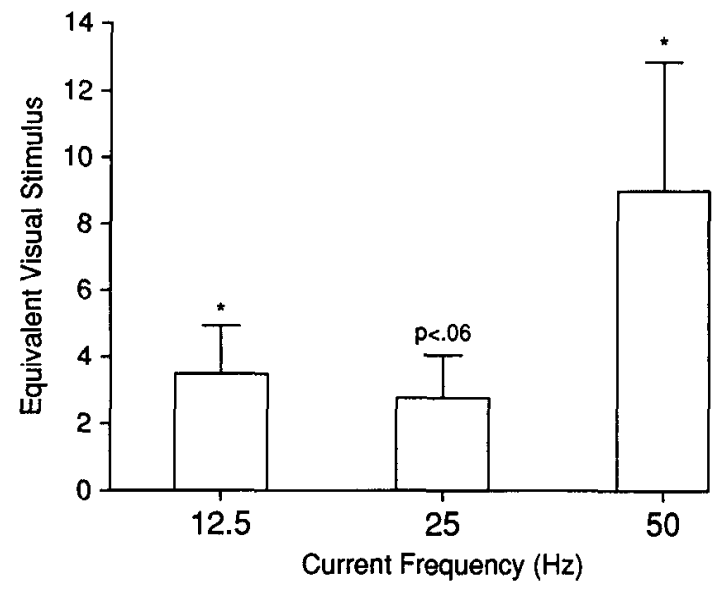

Figure 11. Mean equivalent visual stimulus plotted for each current frequency tested in the single level experiments. Error bars depict 1 SEM, and asterisks denote statistical significance $(p<0.05)$.

motion pathway. The possibility that distinct cortical circuits encode different directions of motion provides a reasonable explanation for the results of the current frequency experiments. As long as direct current spread is limited to a local cluster of neurons with similar directional properties, a directionally specific cortical circuit may be activated. Increases in the amount of transsynaptic activation and the firing rate of neurons within this circuit do not add noise to the neural processes encoding motion direction.

In another series of experiments, the magnitude of the microstimulation effect depended critically on the precise position of the stimulating electrode. In the most striking examples, moving the clcctrode by as little as $100 \mu \mathrm{m}$ transformed "no effect" experiments into remarkably large effects (e.g., Fig. $7 A$ ). This observation suggests that some portion of the stimulation sites that yielded negative results in our previous study (Salzman et al., 1992) may have generated an effect had the electrode been positioned more optimally. At present we know very little about the factors underlying such large effects of electrode position. Proximity of the electrode to layer 4 may account for some of the variability since our prior study suggested that microstimulation of layer 4 generates particularly large behavioral effects (Salzman et al., 1992). Indeed, DeYoe and Doty (Deyoe, 1983) have noted substantial interlaminar differences in the threshold current required to elicit eye movements with microstimulation of striate cortex. On the other hand, the position of a stimulating electrode relative to column boundaries may also influence the outcome. We have very little control of such variables because MT is a folded structure lying deep within the supcrior tcmporal sulcus, and the trajectory of our electrode through MT can make multiple passes through a given cortical layer before exiting from MT. A lack of reliable physiological markers for individual cortical laminae in MT exacerbates this problem. While their origin remains obscure, the effects of electrode position are consistent with the current amplitude experiments in suggesting that neurons near the electrode tip play a primary role in determining the efficacy and functional specificity of the microstimulation signal.

One of the most interesting findings of the present study is that perceptual performance can be influenced robustly by current amplitudes as low as $5 \mu \mathrm{A}$ (for a stimulation frequency of $200 \mathrm{~Hz}$ ) and by stimulation frequencies as low as $25 \mathrm{~Hz}$ (for a 
current amplitude of $10 \mu \mathrm{A}$ ). These figures should be regarded as conservative estimates since measured thresholds may have been lower had we employed more trials. The current 'tmplitude required to elicit effects is comparable to those required for generating eye movements from stimulating the superior colliculus $(1-9 \mu \mathrm{A})$ or the frontal eye fields $(10 \mu \mathrm{A})$ (Schiller and Stryker, 1972; Bruce et al., 1985), or muscle contractions in the hand from stimulating motor cortex $(<10 \mu \mathrm{A})$ (Lemon et al., 1986). The observation that $25 \mathrm{~Hz}$ stimulating frequencies can produce effects must be interpreted with care since the action potentials of cortical neurons do not necessarily follow stimulation pulses in a one-to-one manner (Deyoe, personal communication). The data demonstrate, however, that stimulating frequency need not be beyond the normal physiological response range of MT neurons to elicit behavioral effects.

Together, the data presented in this article and our previous microstimulation study suggest a reasonably consistent set of hypotheses concerning the genesis of microstimulation effects in MT. The amplitude of the stimulating pulses influences the directional specificity of the microstimulation effect: small currents generate directional effects by activating a restricted set of columns with similar preferred directions while large currents yield a nonspecific impairment of performance by activating a more widespread population of columns encoding many directions of motion. The frequency of stimulation modifies the size of the behavioral effect by increasing the firing rate of the stimulated neurons and probably by increasing the size of the transsynaptically activated network. For reasons that are not yet well understood, the precise position of the electrode tip has a major influence on the probability of obtaining a large microstimulation effect. Laminar location and the geometry of direction columns near the electrode tip are likely to account for much of the impact of electrode position. Some of these hypotheses should be testable using techniques such as optical imaging to reveal the pattern of cortical activation resulting from well-defined microstimulation regimes like those employed in this study.

\section{References}

Albright TD (1984) Direction and orientation selectivity of neurons in visual area MT of the macaque. J Neurophysiol 52:1106-1130.

Albright TD, Desimone R, Gross CG (1984) Columnar organization of directionally selective cells in visual area MT of macaques. J Neurophysiol 51:16-31.

Asanuma H, Arnold A, Zarzecki P (1976) Further study on the excitation of pyramidal tract cells by intracortical microstimulation. Exp Brain Res 26:443-461.

Baker JF, Petersen SE, Newsome WT, Allman JM (1981) Visual response properties of neurons in four extrastriate visual areas of the owl monkey (Aotus trivirgatus). J Neurophysiol 45:397-416.

Britten KH, Shadlen MN, Newsome WT, Movshon JA (1992) The analysis of visual motion: a comparison of neuronal and psychophysical performance. J Neurosci 12:4645-4765.

Bruce CJ, Goldberg ME, Bushnell MC, Stanton GB (1985) Primate frontal eye fields. II. Physiological and anatomical correlates of electrically evoked eye movements. J Neurophysiol 54:714-734.

Cox DR (1970) Analysis of binary data. London: Methuen.

Deyoe EA (1983) An investigation in the awake macaque of the threshold for detection of electrical currents applied to striate cortex: psychophysical properties and laminar differences. PhD dissertation, University of Rochester.

Dürsteler MR, Wurtz RH (1988) Pursuit and optokinetic deficits following chemical lesions of cortical areas MT and MST. J Neurophysiol 60:940-965.

Dürsteler MR, Wurtz RH, Newsome WT (1987) Directional pursuit deficits following lesions of the foveal representation within the superior temporal sulcus of the macaque monkey. J Neurophysiol 57: $1262-1287$
Evarts EV (1966) A technique for recording activity of subcortical neurons in moving animals. Electroencephalogr Clin Neurophysiol 24:83-86.

Evarts EV (1968) Methods for recording activity of individual neurons in moving animals. Methods Med Res 11:241-250.

Fuchs AF, Robinson DA (1966) A method for measuring horizontal and vertical eye movement chronically in the monkey. J Appl Physiol $21: 1068-1070$

Gallyas F (1979) Silver staining of myelin by means of physical development. Neurol Res 1:203-209.

Gilbert CD, Wiesel TN (1989) Columnar specificity of intrinsic horizontal and cortico-cortical connections in cat visual cortex. J Neurosci 9:2432-2442.

Jankowska E, Padel Y, Tanaka R (1975) The mode of activation of pyramidal tract cells by intracortical stimuli. J Physiol (Lond) 249: 617-636.

Judge SJ, Richmond BJ, Chu FC (1980) Implantation of magnetic search coils for measurement of eye position: an improved method. Vision Res 20:535-538.

Lemon RN, Mantel GWH, Muir RB (1986) Corticospinal facilitation of hand muscles during voluntary movement in the conscious monkey. J Physiol (Lond) 381:497-527.

Maunsell JHR, Newsome WT (1987) Visual processing in monkey extrastriate cortex. Annu Rev Neurosci 10:363-401.

Maunsell JHR, Van Essen DC (1983) Functional properties of neurons in the middlc tcmporal visual area (MT) of the macaque monkey. I. Selectivity for stimulus direction, speed and orientation. J Neurophysiol 49:1127-1147.

Merigan WH, Pasternak T, Polashenski W, Maunsell JHR (1991) Permanent deficits in speed discrimination after MT/MST lesions in a macaque monkey. Assoc Res Vis Ophthalmol Abstr 32:824.

Murasugi CM, Salzman CD, Newsome WT (1991) Microstimulation of visual area MT during motion discriminations. II. Effects of varying pulse amplitude and frequency. Assoc Res Vis Ophthalmol Abstr 32: 822 .

Newsome WT, Paré EB (1986) MT lesions impair discrimination of direction in a stochastic motion display. Soc Neurosci Abstr 12:1183.

Newsome WT, Paré EB (1988) A selective impairment of motion perception following lesions of the middle temporal visual area (MT). J Ncurosci 8:2201-2211.

Newsome WT, Wurtz RH, Dürsteler MR, Mikami A (1985) Deficits in visual motion processing following ibotenic acid lesions of the middle temporal visual area of the macaque monkey. J Neurosci 5:825-840.

Newsome WT, Britten KH, Movshon JA (1989) Neuronal correlates of a perceptual decision. Nature 341:52-54.

Orbach HS, Felleman DJ, Ribak EN, Van Essen DC (1991) Visualization of cortical connections with voltage sensitive dyes. In: Analysis and modeling of neural systems (Eeckman F, ed), pp 15-28. Norwell, MA: Kluver.

Robinson DA (1963) A method of measuring eye movement using a scleral search coil in a magnetic field. IEEE Trans Biomed Eng 10: 137-145.

Salzman CD, Britten KH, Newsome WT (1990) Cortical microstimulation influences perceptual judgements of motion direction. Nature 346:174-177.

Salzman CD, Murasugi CM, Britten KH, Newsome WT (1992) Microstimulation in visual area MT: effects on direction discrimination performance. J Neurosci 12:2331-2355.

Schiller PH, Stryker M (1972) Single-unit recording and stimulation in superior colliculus of the alert rhesus monkey. J Neurophysiol 35: 915-924.

Siegel RM, Andersen RM (1986) Motion perceptual deficits following ibotenic acid lesions of the middle temporal area (MT) in the behaving rhesus monkey. Soc Neurosci Abstr 12:1183.

Stoney SD Jr, Thompson WD, Asanuma H (1968) Excitation of pyramidal tract cells by intracortical microstimulation: effective extent of stimulating current. J Neurophysiol 31:659-669.

Tootell RBH, Born RT (1991) Architecture of primate area MT. Soc Neurosci Abstr 17:524.

Ts'o DY, Gilbert CD (1988) The organization of chromatic and spatial interactions in the primate striate cortex. J Neurosci 8:1712-1727.

Ts'o DY, Gilbert CD, Wiesel TN (1986) Relationships between horizontal interactions and functional architecture in cat striate cortex as revealed by cross-correlation analysis. J Neurosci 6:1160-1170. 
Vandenbussche E, Saunders RC, Orban GA (1991) MT lesions impair monkey speed discrimination. Assoc Res Vis Ophthalmol Abstr 32: 823.

Van Essen DC, Maunsell JHR, Bixby JL (1981) The middle temporal visual area in the macaque: myeloarchitecture, connections, func- tional properties and topographic representation. J Comp Neurol 199: 293-326.

Zeki SM (1974) Functional organization of a visual area in the posterior bank of the superior temporal sulcus of the rhesus monkey. $J$ Physiol (Lond) 236:549-573. 